\title{
Contribution of the Cholinergic System to Verbal Memory Performance in Mild Cognitive Impairment
}

\author{
Jessica Peter ${ }^{\mathrm{a}, \mathrm{b}, \mathrm{c}, *}$, Jacob Lahr $^{\mathrm{a}, \mathrm{b}}$, Lora Minkovaa, ${ }^{\mathrm{a}, \mathrm{d}}$, Eliza Lauer ${ }^{\mathrm{a}}$, Michel J. Grothe ${ }^{\mathrm{e}}$, Stefan Teipel ${ }^{\mathrm{e}}$, \\ Lena Köstering ${ }^{\mathrm{a}, \mathrm{c}, \mathrm{f}}$, Christoph P. Kaller ${ }^{\mathrm{a}, \mathrm{c}, \mathrm{g}}$, Bernhard Heimbach ${ }^{\mathrm{c}, \mathrm{h}}$, Michael Hüll ${ }^{\mathrm{h}, \mathrm{i}}$, \\ Claus Normann ${ }^{\mathrm{b}}$, Christoph Nissen ${ }^{\mathrm{b}}$, Janine Reis $^{\mathrm{c}}$ and Stefan Klöppel ${ }^{\mathrm{a}, \mathrm{b}, \mathrm{h}}$ \\ ${ }^{a}$ Freiburg Brain Imaging, Faculty of Medicine, University of Freiburg, Germany \\ ${ }^{\mathrm{b}}$ Department of Psychiatry and Psychotherapy, Faculty of Medicine, University of Freiburg, Germany \\ ${ }^{\mathrm{c}}$ Department of Neurology, Faculty of Medicine, University of Freiburg, Germany \\ ${ }^{\mathrm{d}}$ Department of Psychology, Laboratory for Biological and Personality Psychology, University of Freiburg, \\ Germany \\ ${ }^{\mathrm{e}}$ German Centre for Neurodegenerative Diseases (DZNE), Rostock/Greifswald, Germany \\ ${ }^{\mathrm{f}}$ Department of Neuroradiology, Faculty of Medicine, University of Freiburg, Germany \\ $\mathrm{g}^{\mathrm{B}}$ BrainLinks-BrainTools Cluster of Excellence, University of Freiburg, Germany \\ ${ }^{\mathrm{h}}$ Centre for Geriatric Medicine and Gerontology, Faculty of Medicine, University of Freiburg, Germany \\ ${ }^{\mathrm{i}}$ Centre for Psychiatry Emmendingen, Germany
}

Accepted 25 April 2016

\begin{abstract}
Acetylcholine is critically involved in modulating learning and memory function, which both decline in neurodegeneration. It remains unclear to what extent structural and functional changes in the cholinergic system contribute to episodic memory dysfunction in mild cognitive impairment (MCI), in addition to hippocampal degeneration. A better understanding is critical, given that the cholinergic system is the main target of current symptomatic treatment in mild to moderate Alzheimer's disease. We simultaneously assessed the structural and functional integrity of the cholinergic system in 20 patients with MCI and 20 matched healthy controls and examined their effect on verbal episodic memory via multivariate regression analyses. Mediating effects of either cholinergic function or hippocampal volume on the relationship between cholinergic structure and episodic memory were computed. In MCI, a less intact structure and function of the cholinergic system was found. A smaller cholinergic structure was significantly correlated with a functionally more active cholinergic system in patients, but not in controls. This association was not modulated by age or disease severity, arguing against compensational processes. Further analyses indicated that neither functional nor structural changes in the cholinergic system influence verbal episodic memory at the MCI stage. In fact, those associations were fully mediated by hippocampal volume. Although the cholinergic system is structurally and functionally altered in MCI, episodic memory dysfunction results primarily from hippocampal neurodegeneration, which may explain the inefficiency of cholinergic treatment at this disease stage.
\end{abstract}

Keywords: Basal forebrain cholinergic system, mediation, mild cognitive impairment, short afferent inhibition

\section{INTRODUCTION}

Acetylcholine (ACh) plays a critical role in modulating learning and memory processes $[1,2]$, which

${ }^{*}$ Correspondence to: Jessica Peter, PhD, Freiburg Brain Imaging, Department of Psychiatry, University Medical Centre Freiburg, Hauptstraße 5, 79104 Freiburg, Germany. Tel.: +49 761 270 53230; E-mail: jessica.peter@uniklinik-freiburg.de. both decline in healthy aging [3] and (even more) in neurodegenerative diseases [e.g., 4]. Cholinergic neurons can be subdivided into projecting neurons located in the basal forebrain and those located in the brain stem [5], with the basal forebrain cholinergic system (BFCS) representing the predominant cholinergic source of the cerebral cortex and hippocampus [5]. The BFCS includes neurons located 
in the medial septal nucleus, the vertical and horizontal limbs of the diagonal band of Broca, and the nucleus basalis of Meynert (NbM) [6, 7]. Projections of the different subnuclei overlap substantially and mostly reach the hippocampal formation, although some send to neocortical areas, the olfactory bulb and thalamic reticular regions $[2,8,9]$.

In patients with moderate or severe Alzheimer's disease (AD), the BFCS undergoes severe neurofibrillary degeneration and cell loss, which is most pronounced in the NbM [1]. BFCS neurodegeneration, however, may not be a primary event in the pathogenetic cascade of $\mathrm{AD}$, as neuropathological studies did not provide clear evidence for a loss of cholinergic neurons in mild cognitive impairment (MCI, a prodromal stage of $\mathrm{AD}$ ) or mild $\mathrm{AD}$, but altered cholinergic function as indicated by changes in ACh release [see 1 for a review] and an upregulation of ACh synthesis. On the other hand, in an in-vivo MRI study [10], MCI patients $(n=16)$ and AD patients $(n=21)$ showed significantly reduced volumes of the NbM compared to controls, with the degeneration in MCI patients being spatially more restricted compared to those in AD. Furthermore, in a structural MRI study by Grothe and colleagues [11], MCI patients $(n=33)$ exhibited a significant volume reduction in the posterior part of the $\mathrm{NbM}(\mathrm{CH} 4 \mathrm{p})$ compared to elderly controls. Although lacking direct projections to the hippocampus, lower $\mathrm{CH} 4 \mathrm{p}$ volume correlated with worse performance in verbal delayed recall in MCI. Lower volumes in the medial septal nucleus (CH1) and the vertical diagonal band of Broca ( $\mathrm{CH} 2)$, which both send projections to the hippocampus, were correlated with worse global cognitive performance [11]. However, both effects could be driven by overall disease progression rather than being causally dependent, as indicated by a recent study [12] where hypo-metabolisms in a cortical network including the hippocampus was shown to partially mediate the relationship between volume of the BFCS and verbal delayed recall in MCI $(n=132)$.

Complementing work on structural changes of the cholinergic system, another line of research has aimed to assess the integrity of cholinergic function by employing the non-invasive short-latency afferent inhibition (SAI) protocol. SAI is thought to probe an inhibitory circuit in the human cerebral motor cortex [13]. The inhibition refers to the suppression of the amplitude of a motor evoked potential (MEP) induced by a conditioning afferent electrical stimulus prior to transcranial magnetic stimulation (TMS; Fig. 1) [13]. SAI is related to the integrity of cholinergic circuits, as the muscarinic antagonist scopolamine significantly suppressed the inhibition [14], indicating a less active cholinergic system. Furthermore, the inhibitory effect of SAI has been consistently found to be suppressed in AD patients [15] but can be increased for approximately two hours by a single dose of the acetylcholine esterase inhibitor, rivastigmine [16]. SAI effects in MCI were more variable compared to those observed in $A D$, but tended to be suppressed across studies [17-19]. So far, only one study associated SAI with cognition in different subtypes of MCI [19] and found a more pronounced inhibition, indicative of a more active cholinergic system, associated with better verbal long-term memory in amnestic MCI but with worse verbal fluency in non-amnestic MCI.

As previous studies were limited by focusing on either structural or functional properties of the cholinergic system, we aimed to simultaneously examine in-vivo markers of structural (i.e., BFCS volume) and functional (i.e., SAI) changes of the cholinergic system, to assess their interrelation, and to study their effect on verbal episodic memory in MCI [20]. To evaluate whether the examined parameters were altered in MCI, they were also compared to age-, gender-, and education-matched healthy controls. Given the widely distributed neuropathological changes due to $\mathrm{AD}$, it is important to assess the relative contribution of an altered cholinergic system to episodic memory dysfunction in the MCI stage as the cholinergic system is the main target for current symptomatic treatment in mild to moderate $\mathrm{AD}$ [21].

\section{MATERIAL AND METHODS}

\section{Participants}

Twenty patients with MCI $(72.7 \pm 5.6$ years, range: $60-82 ; 10$ female; CDR (sum of boxes) $=0.5$; MMSE $=25.9 \pm 1.9$; Table 1) were recruited from the University Medical Center Freiburg. Cognitive functioning was evaluated using the Consortium to Establish a Registry of Alzheimer's disease (CERAD) neuropsychological battery [22] with German age-, gender-, and education-adjusted norms (http://www.memoryclinic.ch). Participants were diagnosed with MCI if at least one cognitive function was below 1.5 SD according to established criteria $[23,24]$. We did not include patients with a vascular type of MCI or with substantial white matter hyperintensities, as there is a substantial body of literature on the contribution of white matter hyperintensities 

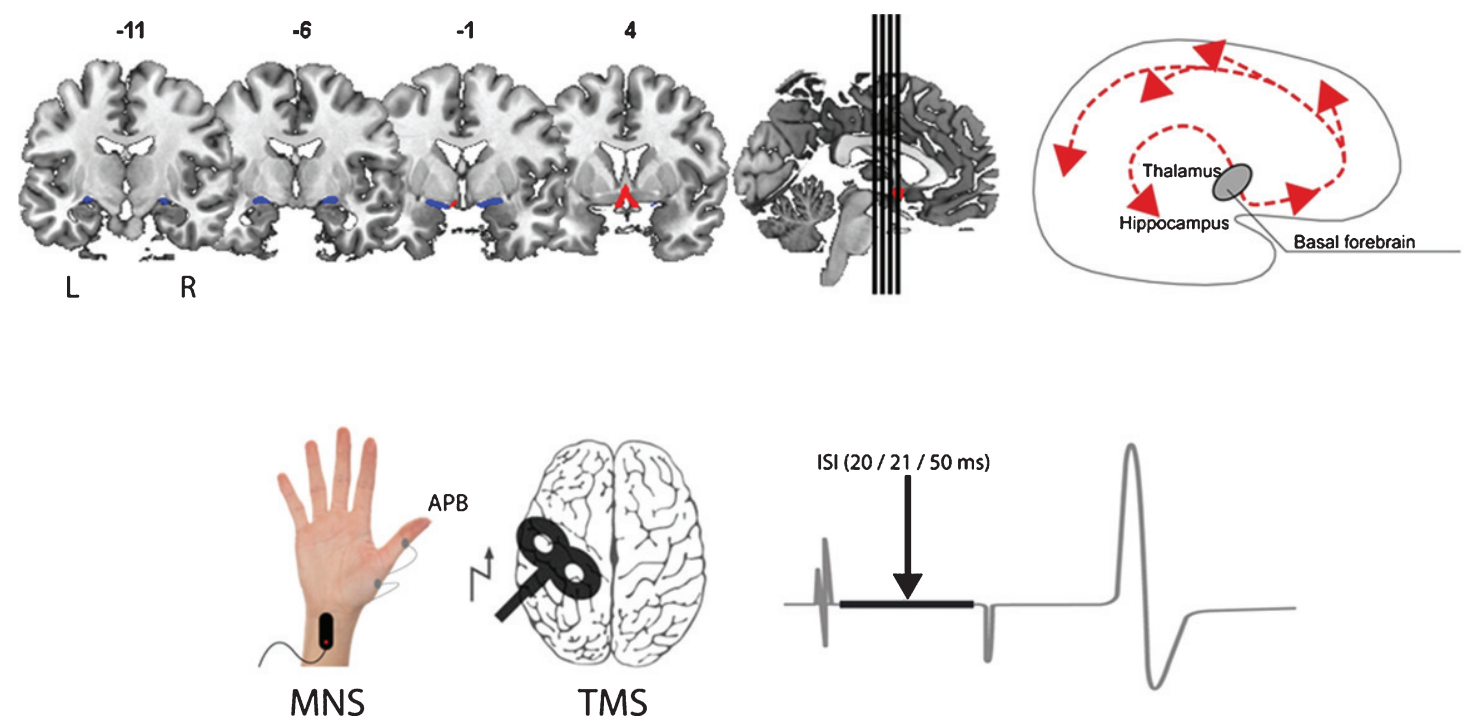

Fig. 1. Illustration of the assessment of structure (top; volume of the basal forebrain - $\mathrm{CH} 1-3$ blue, $\mathrm{CH} 4$ red) and function (bottom; short afferent inhibition) of the cholinergic system in our study. The main cholinergic projections of the basal forebrain are schematically depicted on the top right. MNS, median nerve stimulation; APB, abductor pollicis brevis; TMS, transcranial magnetic stimulation; ISI, interstimulus interval.

Table 1

Socio-demographic data of the sample and group comparison for cholinergic structure and function. Student's $t$-tests were used for group differences if not indicated otherwise

\begin{tabular}{|c|c|c|c|c|c|}
\hline & \multicolumn{2}{|c|}{ Healthy controls } & \multicolumn{2}{|c|}{ MCI } & \multirow[t]{2}{*}{$p$-value } \\
\hline & Mean & SD & Mean & SD & \\
\hline$n(\mathrm{f} / \mathrm{m})$ & $20(6 / 14)$ & & $20(10 / 10)$ & & $0.19\left(\chi^{2}\right.$ test $)$ \\
\hline age (years) & 69.7 & 5.4 & 72.7 & 5.6 & 0.12 \\
\hline years of education & 15.0 & 3.8 & 13.2 & 3.3 & 0.10 \\
\hline BDI-II & 7.4 & 5.7 & 8.1 & 5.8 & 0.67 \\
\hline $\operatorname{MoCA}(0-30)$ & 27.8 & 1.9 & 22.5 & 2.7 & $<0.001$ \\
\hline Word learning, VLMT (0-75) & 52.4 & 9.7 & 32.8 & 9.2 & $<0.001$ \\
\hline Verbal delayed recall, VLMT (0-15) & 10.8 & 3.3 & 3.5 & 2.9 & $<0.001$ \\
\hline Mean of SAI 20 and 21 & 0.54 & 0.19 & 0.69 & 0.33 & 0.07 \\
\hline BFCS (volume in $\mathrm{mm}^{3}$ ) & 637.6 & 53.8 & 557.0 & 68.6 & $<0.001$ \\
\hline Hippocampus (volume in $\mathrm{mm}^{3}$ ) & 7481.2 & 607.1 & 6790.7 & 1074.7 & $<0.05$ \\
\hline
\end{tabular}

SD, standard deviation; MCI, mild cognitive impairment; BDI, Beck's Depression Inventory-II; MoCA, Montreal Cognitive Assessment; VLMT, Verbal Learning and Memory Test; $n$, number; BFCS, basal forebrain cholinergic system; SAI, short afferent inhibition. Basal forebrain and hippocampal volumes indicate the sum of left and right hemispheres.

in the cholinergic system on cognition in neurodegenerative diseases [27-32]. To exclude cases with a predominantly vascular origin of cognitive changes, we excluded those with reported sudden steep decline of cognitive performance, focal neurological signs [33], and major white matter changes observed in the FLAIR image (i.e., three points on the Faszekas scale) [34]. To increase diagnostic certainty, a clinical follow-up was implemented after 12 months.

Twenty healthy controls $(69.7 \pm 5.4$ years, range: 61-81; 14 female; Table 1) were recruited via newspaper advertisement and included if their Montreal Cognitive Assessment (MoCA) score was $\geq 26$ [25] and their Beck's Depression Inventory-II (BDI-II) score was $\leq 13$ [26].
No participant reported a history of severe neurological or psychiatric disease, drug or alcohol abuse, counter-indications to TMS, or neuroactive drug intake. The study was approved by the local Ethics Commission (approval \#227/12) and was conducted according to the Declaration of Helsinki. Written informed consent was obtained from all participants prior to testing.

\section{Genetic analyses}

The $\varepsilon$ 2/3/4 haplotypes of the deoxyribonucleic acid (DNA) samples were analyzed by polymerase chain reaction amplification of a 290-bp frame of exon 3 of the ApoE gene and measurement of base 
mutations contained in codon 112 and 158 by dideoxy sequencing.

\section{Study procedure}

The experiments were conducted on two consecutive afternoons to reduce the effects of fatigue. On the first day of the study, all participants underwent MRI and neuropsychological testing, while TMS was performed on the second day.

\section{Neuropsychological testing}

Verbal memory was assessed using the Verbal Learning and Memory Test (VLMT) [35]. The VLMT is a test of verbal immediate and long-term memory. Participants were asked to listen to a list of 15 words and to reproduce as many words as possible thereafter. There were 5 trials in which the words were presented and retrieved (to assess a rate of learned words, i.e., short-term word learning) and a delayed recall after 30 minutes (without rehearsal).

\section{Short latency afferent inhibition}

TMS was performed using a magnetic stimulator (Magstim 200; Magstim, Whitland, UK) with a figure-of-eight coil. The coil was held over the motor cortex at the optimum scalp position to elicit motor responses in the abductor pollicis brevis muscle. The intensity of the transcranial stimulator was adjusted to result in unconditioned MEP amplitudes of approximately $1 \mathrm{mV}$. TMS pulses were applied at an interval of $6 \mathrm{~s}$ and with a variability of $20 \%$ in order to prevent systematic MEP variability due to expectation.

SAI was applied based on the technique described by Tokimura and colleagues [13]. Conditioning stimuli were single pulses of electrical stimulation applied through bipolar electrodes (cathode proximal, stimulus width $300 \mu \mathrm{s}$ ) to the median nerve at the wrist, using a constant current stimulator (Digitimer DS7; Welwyn, Garden City, UK). Stimulation intensity was adjusted to evoke a visible twitch in the abductor pollicis brevis muscle. The conditioning stimulus to the peripheral nerve preceded the magnetic cortical stimulus by pseudo-randomized inter-stimulus intervals (ISI) of $20 \mathrm{~ms}, 21 \mathrm{~ms}$, and $50 \mathrm{~ms}$ (Fig. 1). The conditions with the ISI of $20 \mathrm{~ms}$ and $21 \mathrm{~ms}$ were used, as Tsutsumi et al. [17] found maximal inhibition with an ISI of $20 \mathrm{~ms}$, while, in Tokimura et al. [13], maximal inhibition was found with an ISI of $21 \mathrm{~ms}$. An ISI of $50 \mathrm{~ms}$ was added as a control condition and was expected to result in a facilitation of the MEP responses [36]. The peak-to-peak MEP amplitude for each ISI was measured (i.e., SAI 20, SAI 21, and SAI 50) and an MEP amplitude ratio was calculated (i.e., SAI ratio, which is the conditioned MEP relative to the unconditioned MEP). The mean MEP amplitude ratio of SAI 20 and SAI 21 was used for further analyses, while SAI 50 served primarily as sanity check. For the MEP amplitude ratio (i.e., SAI ratio), a value of, e.g., 0.75 indicates a reduction of the conditioned MEP-amplitude by $25 \%$ compared to the unconditioned MEP. A smaller SAI ratio thus indicates a stronger inhibitory effect.

\section{Magnetic resonance imaging}

Structural MRI was acquired using a threedimensional Magnetization Prepared Rapid Acquisition Gradient Echo (MPRAGE) sequence and a 12-channel standard head coil using a $3 \mathrm{~T}$ scanner (Tim Trio; Siemens, Erlangen, Germany). The parameters applied were as follows: flip angle of $12^{\circ}$, repetition time (TR) of $2200 \mathrm{~ms}$, echo time (TE) of $2.15 \mathrm{~s}$, matrix size of $256 \times 256 \times 176$, and slice thickness of $1 \mathrm{~mm}$. The entire MRI session was 45 minutes. No participant withdrew from the study due to claustrophobia. Besides structural MRI (i.e., T1), T2, FLAIR (TE $388 \mathrm{~ms}$, TR $5000 \mathrm{~ms}$, matrix size $256 \times 256 \times 260$, slice thickness $1 \mathrm{~mm}$ ), DTI, and resting state fMRI were acquired. Results from the other MRI contrasts may be reported elsewhere. MRI data were preprocessed using Statistical Parametric Mapping (SPM12, Welcome Trust Center for Neuroimaging) and the VBM8 toolbox (http://dbm.neuro.uni-jena.de/vbm/) implemented in Matlab R2013b (MathWorks, Natick, MA, USA). MRI scans were segmented into gray matter (GM), white matter, and cerebrospinal fluid and highdimensionally registered to Montreal Neurological Institute (MNI) standard space using the VBM8 toolbox. GM voxel values were modulated to account for the volumetric differences introduced by the high-dimensional warps but not for an initial affine registration step that served to account for global differences in brain size. GM volumes of the BF cholinergic nuclei were automatically extracted by summing up the modulated GM voxel values within the respective regions (see below).

\section{Basal forebrain cholinergic system volumetry}

A stereotactic atlas of the BFCS based on combined postmortem MRI and histological examination 
of cholinergic nuclei was used [37] (Fig. 1). As it is based on the histological examination of a single brain specimen and may not generalize across individuals, the coordinates (i.e., centers of gravity) of the cytoarchitectonic areas published by Zaborszky et al. [38] and a mask of the BFCS obtained from the Anatomy Toolbox (version 2.1) were used to replicate the results. As projections of the BFCS' subnuclei overlap substantially, we did not further subdivide the BFCS, comparable to previous studies [39, 40].

\section{Hippocampal volumetry}

GM volumes of the left and right hippocampus were extracted automatically using FreeSurfer 5.3 [41] (http://surfer.nmr.mgh.harvard.edu) followed by visual quality control. FreeSurfer analyses were manually checked by two raters and compared to an anatomical atlas. An agreement between raters was sought on all cases with low segmentation quality. We did not perform a formal inter-rater reliability analysis because ratings were not done completely independently. The hippocampus is strongly associated with changes in episodic memory performance and atrophies at an annual rate of approximately $3 \%$ [42]. The normalized sum of left and right hippocampal volume was calculated for each individual, using residuals from a linear regression between hippocampal volume (y) and total intracranial volume (x) as described by Jack et al. [43].

\section{Statistical analyses}

Statistical analysis was performed using the SPSS software package (version 21.0; IBM Inc.; Armonk, USA) and a critical $p<0.05$ for statistical significance. Parametric tests were used unless stated otherwise (all Kolmogorov-Smirnoff tests: $p>0.7$ ). We computed bivariate correlations between structural and functional markers of the cholinergic system and cognitive outcome. To assess within and between group differences induced by SAI-TMS, a two-bytwo repeated-measures ANOVA (rmANOVA) with the unconditioned MEP and the mean of the raw MEP following SAI20 and SAI21 was calculated. As a plausibility check, a separate two-by-two rmANOVA was calculated for the raw MEP following SAI50.

\section{Mediation effects}

To gain further insight into the interrelation between structure and function of the BFCS, and the clinical consequences of these relations in MCI, the SPSS PROCESS macro [version 2.10; 44] was used to estimate direct and indirect effects of a predictor on an outcome (mediation analyses, see below), with bias-corrected $95 \%$ bootstrap confidence intervals for the indirect effects. Mediation analyses test the assumption that the bivariate relationship between a predictor variable $\mathrm{X}$ and an outcome variable $\mathrm{Y}$ is mediated by a third variable $M$, with $M$ assumed to be affected by $\mathrm{X}$ and in turn to predict $\mathrm{Y}$, thereby contributing to the effect of $\mathrm{X}$ on $\mathrm{Y}$.

We computed mediation analyses (Model 4 of the PROCESS macro) in MCI, to assess if the strength of the SAI ratio mediated the relationship between BFCS volume and word learning or delayed recall, respectively. To address if previously reported associations between BFCS volume and verbal memory in MCI were driven by neurodegeneration of the hippocampus, we additionally assessed if hippocampal volume serves as a mediator for the association between BFCS volume and word learning or verbal delayed recall, respectively.

We used BFCS volume as a focal predictor X, verbal delayed recall (or word learning, respectively) as outcome variable Y and strength of SAI (or hippocampal volume, respectively) as the mediator $\mathrm{M}$ (Figs. 2A, 3A). The analysis tests the possibility (with $95 \%$ confidence) of the indirect effect (the mediating effect) to be zero, which would indicate that there is no mediation. Bootstrapping (50.000 iterations) was preferred over the Sobel test because it provides greater statistical power in testing the significance of the indirect effect [44].

\section{RESULTS}

MCI patients showed worse cognitive performance compared to healthy controls. They had significantly lower MoCA scores, and learned and retrieved significantly fewer words (Table 1). Additionally, they showed significantly smaller BFCS and hippocampal volumes compared to healthy controls (Table 1), but no significant difference in brain size could be observed $\left(\mathrm{t}_{(38)}=1.463, p=0.15\right)$.

The MEP following SAI20 was $0.57 \pm 0.23$ in healthy controls and $0.71 \pm 0.34$ in patients with MCI, while for SAI21 the MEP was $0.52 \pm 0.20$ in healthy controls and $0.69 \pm 0.35$ in patients with MCI, respectively. The rmANOVA-based analyses of the mean SAI20/21 effect revealed a significant SAI effect $\left(\mathrm{F}_{(2,76)}=38.44, p<0.001\right)$, no significant 

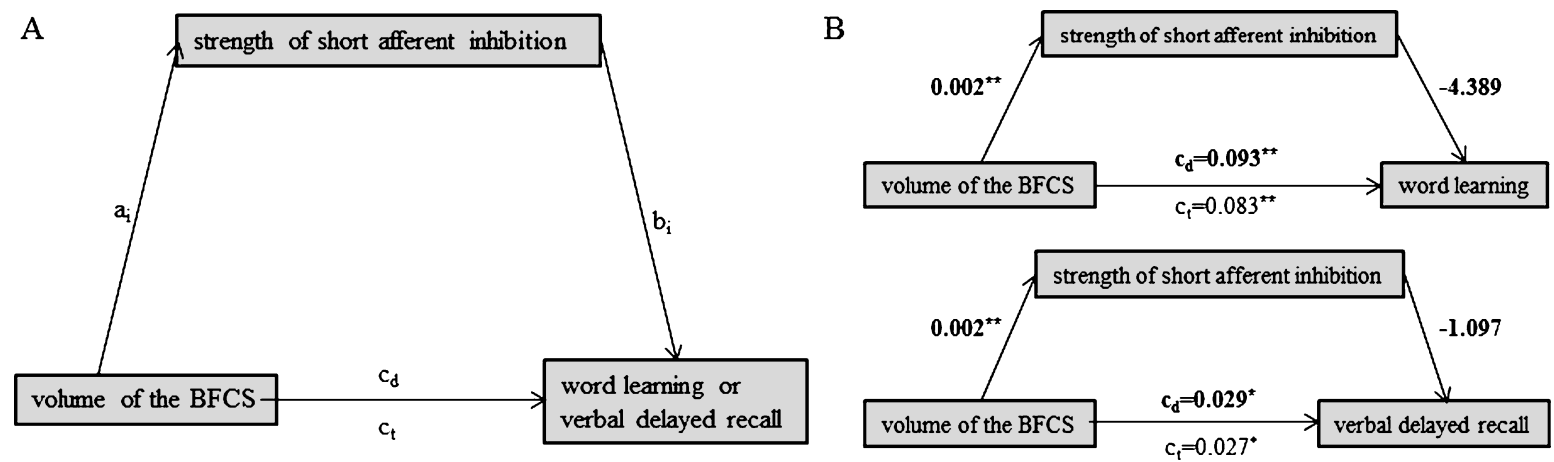

Fig. 2. Schematic of mediation (A) and the calculated mediating effects of short afferent inhibition strength on the relationship between volume of the BFCS and word learning (B, top) or verbal delayed recall (B, bottom). All path weights refer to standardized regression coefficients; $c_{t}$ denotes the total effect and $c_{d}$ the direct effect of the predictor on the outcome variable. $*$ Significant at $\alpha=0.05, * * \alpha=0.01$, ${ }^{* * *} \alpha<0.001$, two-tailed.
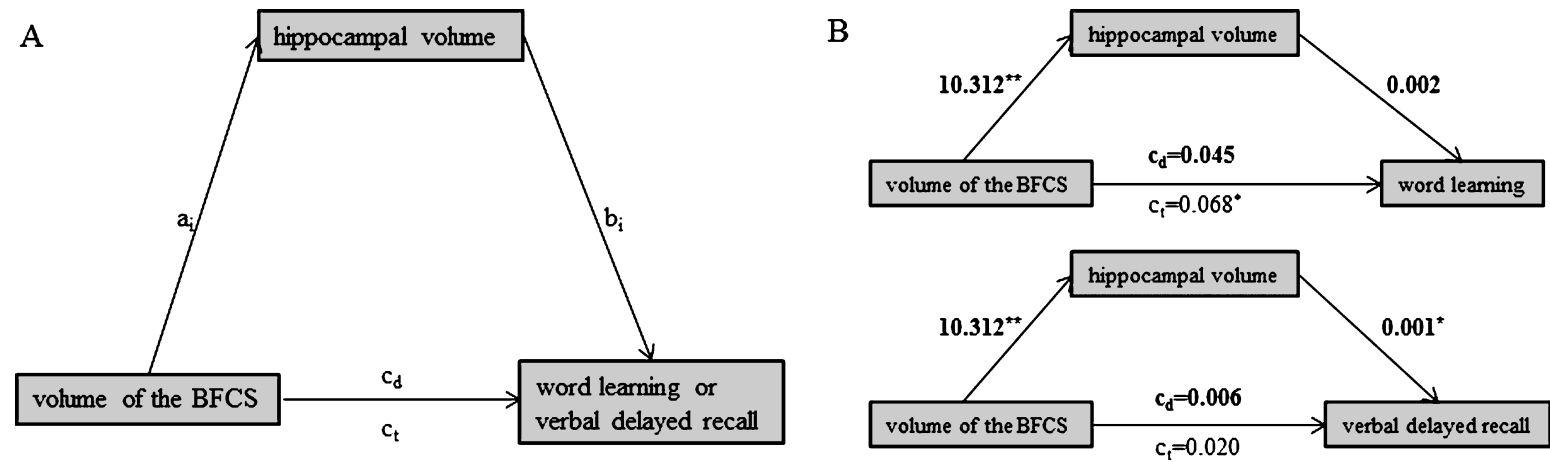

Fig. 3. Schematic of mediation (A) and the calculated mediating effects of hippocampal volume on the relationship between BFCS and word learning ( $\mathrm{B}$, top) or verbal delayed recall $\left(\mathrm{B}\right.$, bottom). All path weights refer to standardized regression coefficients; $\mathrm{c}_{\mathrm{t}}$ denotes the total effect and $\mathrm{c}_{\mathrm{d}}$ the direct effect of the predictor on the outcome variable. ${ }^{*}$ Significant at $\alpha=0.05,{ }^{* *} \alpha=0.01,{ }^{* * *} \alpha<0.001$, two-tailed.

group effect $\left(\mathrm{F}_{(1,38)}=0.06, p=0.81\right)$ and no significant interaction $\left(\mathrm{F}_{(2,76)}=0.54, p=0.46\right)$. The same analyses based on SAI50 showed a significant facilitation $\left(\mathrm{F}_{(1,38)}=4.45, p<0.05\right)$ of SAI $(\mathrm{MEP}$ following SAI50 was $1.10 \pm 0.54$ in healthy controls and $1.24 \pm 0.42$ in patients with $\mathrm{MCI})$, no effect of group $\left(\mathrm{F}_{(1,38)}=0.09, p=0.76\right)$ and no interaction $\left(\mathrm{F}_{(1,38)}=0.44, p=0.51\right)$.

\section{Genetic analyses}

Genetic data were available from $20 \mathrm{HCs}$ and 19 MCIs. In HC, the distribution of the ApoE gene was the following: $\varepsilon 2 / \varepsilon 2=2, \varepsilon 2 / \varepsilon 3=2, \varepsilon 3 / \varepsilon 3=6$, $\varepsilon 3 / \varepsilon 4=10$. There was no $\mathrm{HC}$ with $\varepsilon 4 / \varepsilon 4$. In the MCI group, the distribution was $\varepsilon 3 / \varepsilon 3=9, \varepsilon 3 / \varepsilon 4=7$, $\varepsilon 4 / \varepsilon 4=3$. There was no MCI patient with $\varepsilon 2 / \varepsilon 2$ or $\varepsilon 2 / \varepsilon 3$. Within the MCI group, carriers of the $\varepsilon 4$ allele had significantly smaller volumes of the hippocampus (non-carrier of $\varepsilon 4754.3 \pm 137.5$, carrier of $\varepsilon 4$ $645.8 \pm 44.61 ; p<0.05)$. On a statistical trend level, carriers of $\varepsilon 4$ showed reduced BFCS volumes (noncarrier of $\varepsilon 4584.1 \pm 86.2$, carrier of $\varepsilon 4531.5 \pm 43.2$; $p=0.10$ ) and lower MoCA scores (non-carrier of $\varepsilon 4$ $23.8 \pm 1.6$, carrier of $\varepsilon 421.4 \pm 3.2 ; p=0.06$ ). We did not find other significant differences (e.g. for verbal delayed recall).

\section{Bivariate correlations between structure and function of the cholinergic system}

In MCI, smaller volumes of the BFCS were associated with a more active cholinergic system as indexed by a stronger inhibitory effect of the SAI protocol on the MEP $(r=0.49, p<0.05)$. Similar correlations were absent in $\mathrm{HC}(r=0.07, p=0.76$; Fig. 4). No other significant correlation was found for SAI (Table 2).

Volume of the BFCS correlated significantly positively with volume of the hippocampus $(r=0.59$, $p<0.01)$, verbal word learning $(r=0.45, p<0.05)$, 


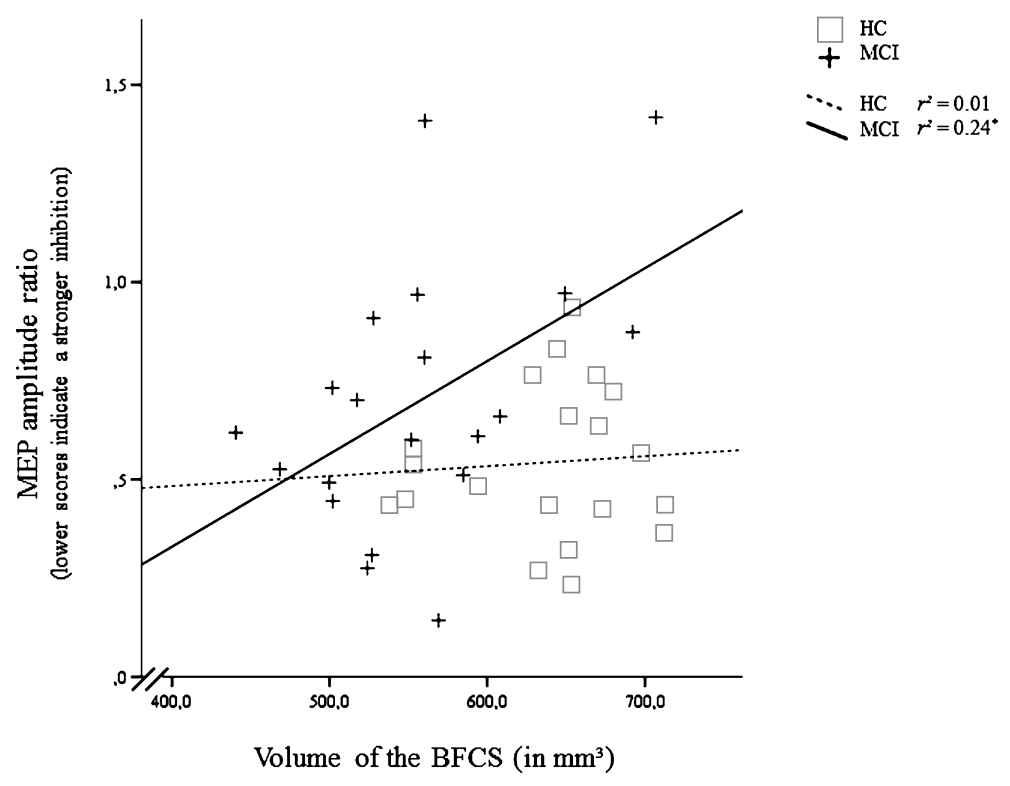

Fig. 4. Correlation of the inhibitory effect of the short afferent inhibition (SAI) protocol (Motor Evoked Potential after, relative to before SAI) and volume of the basal forebrain cholinergic system (BFCS) in mild cognitive impairment (MCI) and healthy controls (HC). For the MEP amplitude ratio, smaller values indicate a stronger inhibition. *Significant at $\alpha=0.05$, two-tailed.

Table 2

Correlation matrix for structural and functional integrity of the basal forebrain cholinergic system and age in MCI

\begin{tabular}{lccccr}
\hline & SAI ratio & Volume of the BFCS & Verbal delayed recall & Word learning & Age \\
\hline SAI ratio & 1.00 & & & & \\
Volume of the BFCS & $0.49^{*}$ & 1.00 & & & \\
Verbal delayed recall & 0.13 & $0.49^{*}$ & 1.00 & 1.00 & \\
Word learning & 0.11 & $0.45^{*}$ & $0.72^{* * *}$ & -0.29 & 1.00 \\
Age & -0.03 & -0.28 & -0.20 & \\
\hline
\end{tabular}

MCI, mild cognitive impairment; BFCS, basal forebrain cholinergic system; SAI, short afferent inhibition (mean of SAI20 and SAI21). ${ }^{*} p<0.05 ;{ }^{* *} p<0.01 ;{ }^{* * *} p<0.001$.

and verbal delayed recall $(\mathrm{r}=0.49, p<0.05)$. Volume of the hippocampus correlated significantly positively with verbal delayed recall $(\mathrm{r}=0.57, p<0.01)$ and to a trend with word learning $(\mathrm{r}=0.43, p$ $=0.06$ ).

\section{Mediating role of SAI or hippocampal volume}

We found no evidence for a mediating role of SAI on the association between BFCS volume and either word learning (indirect effect: $\mathrm{b}=-0.010,95 \%$ bias corrected CI $[-0.015,0.005])$ or verbal delayed recall (indirect effect: $b=-0.002,95 \%$ bias corrected CI $[-0.004,0.026])$ (Fig. 2B). Likewise, hippocampal volume did not mediate the association between BFCS volume and word learning (Fig. 3B; indirect effect $b=0.023,95 \%$ bias corrected CI $[-0.026$, 0.080]).
However, hippocampal volume fully mediated the effect of BFCS volume on verbal delayed recall, as we found a significant indirect effect of hippocampal volume on the relationship between BFCS and verbal delayed recall $(b=0.015,95 \%$ bias corrected CI [0.002, 0.033], while the direct effect of BFCS volume on verbal delayed recall was not significant $(b=0.001, p=0.61)$ (Fig. 3B).

We found a left-volume-less-than-right asymmetry pattern that has been reported earlier [e.g., 45]. In patients with MCI, left hippocampal volume was $3296.0 \pm 527.6$, while volume of the right hippocampus was $3494.6 \pm 546.7$. However, left and right hippocampi correlated significantly $(\mathrm{r}=0.68$, $p<0.001$; 'large' according to [46]). Nevertheless, we repeated our analyses using left hippocampal volume only (as the left hippocampus is particularly related to verbal memory). Again, the association 
between volume of the BFCS and verbal delayed recall was completely driven by (left) hippocampal volume.

\section{DISCUSSION}

The aim of this study was to simultaneously examine structural and functional properties of the cholinergic system as well as to explore their effects on episodic memory performance in patients with MCI.

Comparable to previous studies, TMS with the SAI protocol resulted in a significant inhibition of the MEP at an ISI of both 20 and $21 \mathrm{~ms}$ in MCI patients and healthy controls $[13,17]$, while MEPs at an ISI of $50 \mathrm{~ms}$ showed a facilitation [36]. When comparing groups, the inhibition of the MEP amplitude (i.e., SAI ratio) tended to be suppressed in MCI, which is well in line with previous studies [13, 17-19], although between-group differences reached significance in some of them [17, 19]. Similar to previous studies, patients with MCI showed both significantly smaller BFCS [10, 11] and hippocampal volumes [e.g., 47] compared to healthy controls.

Although patients with MCI tended to show a suppressed SAI ratio, we found a significantly positive correlation between SAI ratio and volume of the BFCS, indicating that individuals with smaller volumes of the BFCS showed a relatively more pronounced inhibition of the MEP reflecting a more active cholinergic system (Fig. 4). The correlation remained significant when controlling for hippocampal volume or MoCA (as an index of disease severity), or age. The association could be the in-vivo correlate of the complex reorganizations of the cholinergic system observed in histopathological studies [1]. Those include an upregulation of ACh synthesis which could be compensatory in nature. However, our findings argue against a compensatory nature of these changes: We neither found the correlation between SAI ratio and BFCS volume to be modulated by age or disease severity (MoCA, hippocampal volume) nor did the SAI ratio mediate the association between BFCS volume and memory performance. Although we replicated existing studies by demonstrating a significantly positive correlation between verbal delayed recall and BFCS volume in MCI [11], this association was fully mediated by hippocampal volume in our study. This mediating effect was also observed when restricting the analysis to the left hippocampus, which has been associated with verbal delayed recall both in the healthy elderly and in patients with MCI $[48,49]$. With the exception of a positive association between short-term learning and BFCS volume, which could be driven by the effect of the cholinergic system on attentional processes [50, 51], we conclude that neither functional nor structural changes in the cholinergic system influence verbal memory dysfunction in MCI. This statement is supported by histological studies revealing that cognitive deficits are not evident before at least $30 \%$ of the BFCS cells have degenerated [5] and our MCI sample showed only around $15 \%$ volume loss in the BFCS compared to HC. Our findings therefore indicate that changes in the cholinergic system do not drive episodic memory dysfunction in MCI, which may explain the lack of response to cholinergic treatment that has been found both in earlier studies [e.g., 52] and in a recent meta-analysis [53]. Our findings may also provide an explanation why basal forebrain volume did not significantly predict response to cholinergic treatment in a double-blind, randomized, placebocontrolled trial in MCI $(n=215)$ over 12 months [54]. Treated patients did not differ from controls in their rate of decline, although the patients treated with donepezil showed a significantly less shrinkage of the hippocampus [55].

\section{Limitations}

Although multimodal, our approach does not capture all contributing factors. The SAI effect, for example, does not exclusively probe the cholinergic system, as it is also modulated by the dopaminergic system [56, 57]. Similarly, although the great majority of neurons in the BFCS are cholinergic (e.g., $90 \%$ in the $\mathrm{NbM} ; 42$ ), our volumetric approach does not allow us to identify the degenerating population of neurons and neither are we able to distinguish between death of projecting neurons or changes in synaptic density [1]. Although the main analyses were performed with a BFCS mask derived from a single subject, results were validated when repeating our analysis with the coordinates (i.e., centers of gravity) of the cytoarchitectonic areas published by Zaborszky et al. [38] and a BFCS mask obtained from the Anatomy Toolbox (version 2.1).

In summary, we found bivariate associations between volume of the BFCS and strength of SAI but no mediating effect of SAI on the association between BFCS and verbal memory performance. The relationship between BFCS volume and verbal long-term memory was completely driven by hippocampal 
atrophy. Our findings therefore indicate that changes in the cholinergic system do not drive episodic memory dysfunction in MCI. This may explain the lack of response to cholinergic treatment at this stage of the disease.

\section{ACKNOWLEDGMENTS}

The authors would like to thank Simon Eickhoff and Laszlo Zaborsky for their help with the BFCS masks. LK is supported by scholarship funds from the State Graduate Funding Program of BadenWürttemberg, Germany.

Authors' disclosures available online (http://jalz.com/manuscript-disclosures/16-0273r2).

\section{REFERENCES}

[1] Schliebs R, Arendt T (2011) The cholinergic system in aging and neuronal degeneration. Behav Brain Res 221, 555-563.

[2] Gratwicke J, Kahan J, Zrinzo L, Hariz M, Limousin P, Foltynie T, Jahanshahi M (2013) The nucleus basalis of Meynert: A new target for deep brain stimulation in dementia? Neurosci Biobehav Rev 37, 2676-2688.

[3] Nyberg L, Lövdén M, Riklund K, Lindenberger U, Bäckman L (2012) Memory aging and brain maintenance. Trends Cogn Sci 16, 292-305.

[4] Irish M, Hornberger M (2015) Episodic memory in neurodegenerative disorders. In The Wiley Handbook on the Cognitive Neuroscience of Memory, Addis DR, Barense M, Duarte A, eds. John Wiley \& Sons, Ltd, pp. 415-433.

[5] Schliebs R, Arendt T (2006) The significance of the cholinergic system in the brain during aging and in Alzheimer's disease. J Neural Transm 113, 1625-1644.

[6] Zaborszky L, Pang K, Somogyi J, Nadasdy Z, Kallo I (1999) The basal forebrain corticopetal system revisited. Ann NY Acad Sci 877, 339-367.

[7] Zaborszky L, Csordas A, Mosca K, Kim J, Gielow MR, Vadasz C, Nadasdy Z (2015) Neurons in the basal forebrain project to the cortex in a complex topographic organization that reflects corticocortical connectivity patterns: An experimental study based on retrograde tracing and 3D reconstruction. Cereb Cortex 25, 118-137.

[8] Gaykema RPA, Luiten PGM, Nyakas C, Traber J (1990) Cortical projection patterns of the medial septum-diagonal band complex. J Comp Neurol 293, 103-124.

[9] Hof PR, Mobbs CV (2001) Functional Neurobiology of Aging, Academic Press.

[10] Teipel SJ, Meindl T, Grinberg L, Grothe M, Cantero JL, Reiser MF, Möller H-J, Heinsen H, Hampel H (2011) The cholinergic system in mild cognitive impairment and Alzheimer's disease: An in vivo MRI and DTI study. Hum Brain Mapp 32, 1349-1362.

[11] Grothe M, Zaborszky L, Atienza M, Gil-Neciga E, Rodriguez-Romero R, Teipel SJ, Amunts K, SuarezGonzalez A, Cantero JL (2010) Reduction of basal forebrain cholinergic system parallels cognitive impairment in patients at high risk of developing Alzheimer's disease. Cereb Cortex 20, 1685-1695.
[12] Grothe MJ, Heinsen H, Amaro E, Grinberg LT, Teipel SJ, Alzheimer's Disease Neuroimaging Initiative (2016) Cognitive correlates of basal forebrain atrophy and associated cortical hypometabolism in mild cognitive impairment. Cereb Cortex 26, 2411-2426.

[13] Tokimura H, Di Lazzaro V, Tokimura Y, Oliviero A, Profice P, Insola A, Mazzone P, Tonali P, Rothwell JC (2000) Short latency inhibition of human hand motor cortex by somatosensory input from the hand. J Physiol 523(Pt 2), 503-513.

[14] Di Lazzaro V, Oliviero A, Profice P, Pennisi MA, Di Giovanni S, Zito G, Tonali P, Rothwell JC (2000) Muscarinic receptor blockade has differential effects on the excitability of intracortical circuits in the human motor cortex. Exp Brain Res 135, 455-461.

[15] Freitas C, Mondragón-Llorca H, Pascual-Leone A (2011) Noninvasive brain stimulation in Alzheimer's disease: Systematic review and perspectives for the future. Exp Gerontol 46, 611-627.

[16] Di Lazzaro V, Oliviero A, Pilato F, Saturno E, Dileone M, Marra C, Ghirlanda S, Ranieri F, Gainotti G, Tonali P (2005) Neurophysiological predictors of long term response to AChE inhibitors in AD patients. J Neurol Neurosurg Psychiatry 76, 1064-1069.

[17] Tsutsumi R, Hanajima R, Hamada M, Shirota Y, Matsumoto H, Terao Y, Ohminami S, Yamakawa Y, Shimada H, Tsuji S, Ugawa Y (2012) Reduced interhemispheric inhibition in mild cognitive impairment. Exp Brain Res 218, 21-26.

[18] Sakuma K, Murakami T, Nakashima K (2007) Short latency afferent inhibition is not impaired in mild cognitive impairment. Clin Neurophysiol 118, 1460-1463.

[19] Nardone R, Bergmann J, Christova M, Caleri F, Tezzon F, Ladurner G, Trinka E, Golaszewski S (2012) Short latency afferent inhibition differs among the subtypes of mild cognitive impairment. J Neural Transm 119, 463-471.

[20] Ribeiro F, Guerreiro M, De Mendonça A (2007) Verbal learning and memory deficits in Mild Cognitive Impairment. J Clin Exp Neuropsychol 29, 187-197.

[21] Doody RS (2003) Current treatments for Alzheimer's disease: Cholinesterase inhibitors. J Clin Psychiatry 64(Suppl 9), 11-17.

[22] Morris JC, Heyman A, Mohs RC, Hughes JP, van Belle G, Fillenbaum G, Mellits ED, Clark C (1989) The Consortium to Establish a Registry for Alzheimer's Disease (CERAD). Part I. Clinical and neuropsychological assessment of Alzheimer's disease. Neurology 39, 1159-1165.

[23] Petersen RC, Smith GE, Waring SC, Ivnik RJ, Tangalos EG, Kokmen E (1999) Mild cognitive impairment: Clinical characterization and outcome. Arch Neurol 56, 303-308.

[24] Albert MS, DeKosky ST, Dickson D, Dubois B, Feldman HH, Fox NC, Gamst A, Holtzman DM, Jagust WJ, Petersen RC, Snyder PJ, Carrillo MC, Thies B, Phelps CH (2011) The diagnosis of mild cognitive impairment due to Alzheimer's disease: Recommendations from the National Institute on Aging-Alzheimer's Association workgroups on diagnostic guidelines for Alzheimer's disease. Alzheimers Dement 7, 270-279.

[25] Nasreddine ZS, Phillips NA, Bédirian V, Charbonneau S, Whitehead V, Collin I, Cummings JL, Chertkow H (2005) The Montreal Cognitive Assessment, MoCA: A brief screening tool for mild cognitive impairment. J Am Geriatr Soc 53, 695-699.

[26] Beck AT, Ward CH, Mendelson M, Mock J, Erbaugh J (1961) An inventory for measuring depression. Arch Gen Psychiatry 4, 561-571. 
[27] Muir RT, Lam B, Honjo K, Harry RD, McNeely AA, Gao F-Q, Ramirez J, Scott CJM, Ganda A, Zhao J, Zhou XJ, Graham SJ, Rangwala N, Gibson E, Lobaugh NJ, Kiss A, Stuss DT, Nyenhuis DL, Lee B-C, Kang Y, Black SE (2015) Trail making test elucidates neural substrates of specific poststroke executive dysfunctions. Stroke 46, 2755-2761.

[28] Park H-E, Park I-S, Oh Y-S, Yang D-W, Lee K-S, Choi H-S, Ahn K-J, Kim J-S (2015) Subcortical whiter matter hyperintensities within the cholinergic pathways of patients with dementia and parkinsonism. J Neurol Sci 353, 44-48.

[29] Swartz RH, Sahlas DJ, Black SE (2003) Strategic involvement of cholinergic pathways and executive dysfunction: Does location of white matter signal hyperintensities matter? J Stroke Cerebrovasc Dis 12, 29-36.

[30] Kim H-J, Moon W-J, Han S-H (2013) Differential cholinergic pathway involvement in Alzheimer's disease and subcortical ischemic vascular dementia. J Alzheimers Dis 35, 129-136.

[31] McNeely AA, Ramirez J, Nestor SM, Zhao J, Gao F, Kiss A, Stuss DT, Black SE (2015) Cholinergic subcortical hyperintensities in Alzheimer's disease patients from the Sunnybrook Dementia Study: Relationships with cognitive dysfunction and hippocampal atrophy. $J$ Alzheimers Dis 43, 785-796.

[32] Bohnen NI, Muller MLTM, Kuwabara H, Constantine GM, Studenski SA (2009) Age-associated leukoaraiosis and cortical cholinergic deafferentation. Neurology 72, 1411-1416.

[33] McKhann GM, Knopman DS, Chertkow H, Hyman BT, Jack CR Jr, Kawas CH, Klunk WE, Koroshetz WJ, Manly JJ, Mayeux R, Mohs RC, Morris JC, Rossor MN, Scheltens P, Carrillo MC, Thies B, Weintraub S, Phelps $\mathrm{CH}$ (2011) The diagnosis of dementia due to Alzheimer's disease: Recommendations from the National Institute on Aging-Alzheimer's Association workgroups on diagnostic guidelines for Alzheimer's disease. Alzheimers Dement 7, 263-269.

[34] Fazekas F, Chawluk JB, Alavi A, Hurtig HI, Zimmerman RA (1987) MR signal abnormalities at $1.5 \mathrm{~T}$ in Alzheimer's dementia and normal aging. AJR Am J Roentgenol 149, 351356.

[35] Helmstaedter C, Lendt M, Lux S (2001) Verbaler Lern- und Merkfähigkeitstest, Hogrefe, Göttingen.

[36] Devanne H, Degardin A, Tyvaert L, Bocquillon P, Houdayer E, Manceaux A, Derambure P, Cassim F (2009) Afferentinduced facilitation of primary motor cortex excitability in the region controlling hand muscles in humans. Eur $J$ Neurosci 30, 439-448.

[37] Kilimann I, Grothe M, Heinsen H, Alho EJL, Grinberg L, Amaro E, Dos Santos GAB, da Silva RE, Mitchell AJ, Frisoni GB, Bokde ALW, Fellgiebel A, Filippi M, Hampel H, Klöppel S, Teipel SJ (2014) Subregional basal forebrain atrophy in Alzheimer's disease: A multicenter study. J Alzheimers Dis 40, 687-700.

[38] Zaborszky L, Hoemke L, Mohlberg H, Schleicher A, Amunts K, Zilles K (2008) Stereotaxic probabilistic maps of the magnocellular cell groups in human basal forebrain. Neuroimage 42, 1127-1141.

[39] Grothe M, Heinsen H, Teipel SJ (2012) Atrophy of the cholinergic basal forebrain over the adult age range and in early stages of Alzheimer's disease. Biol Psychiatry 71, 805-813.

[40] Grothe M, Heinsen H, Teipel S (2013) Longitudinal measures of cholinergic forebrain atrophy in the transition from healthy aging to Alzheimer's disease. Neurobiol Aging 34, 1210-1220.
[41] Fischl B, Salat DH, Busa E, Albert M, Dieterich M, Haselgrove C, van der Kouwe A, Killiany R, Kennedy D, Klaveness S, Montillo A, Makris N, Rosen B, Dale AM (2002) Whole brain segmentation: Automated labeling of neuroanatomical structures in the human brain. Neuron 33, 341-355.

[42] Jack CR, Petersen RC, Xu Y, O'Brien PC, Smith GE, Ivnik RJ, Boeve BF, Tangalos EG, Kokmen E (2000) Rates of hippocampal atrophy correlate with change in clinical status in aging and AD. Neurology 55, 484-489.

[43] Jack CR, Knopman DS, Weigand SD, Wiste HJ, Vemuri P, Lowe V, Kantarci K, Gunter JL, Senjem ML, Ivnik RJ, Roberts RO, Rocca WA, Boeve BF, Petersen RC (2012) An operational approach to National Institute on AgingAlzheimer's Association criteria for preclinical Alzheimer disease. Ann Neurol 71, 765-775.

[44] Hayes AF (2013) Introduction to mediation, moderation, and conditional process analysis: A regression-based approach, The Guilford Press, New York.

[45] Shi F, Liu B, Zhou Y, Yu C, Jiang T (2009) Hippocampal volume and asymmetry in mild cognitive impairment and Alzheimer's disease: Meta-analyses of MRI studies. Hippocampus 19, 1055-1064.

[46] Cohen J (1988) Statistical power analysis for the behavioral sciences, L. Erlbaum Associates, Hillsdale, N.J.

[47] Tae WS, Kim SS, Lee KU, Nam E-C, Kim KW (2008) Validation of hippocampal volumes measured using a manual method and two automated methods (FreeSurfer and IBASPM) in chronic major depressive disorder. Neuroradiology 50, 569-581.

[48] Chen KHM, Chuah LYM, Sim SKY, Chee MWL (2010) Hippocampal region-specific contributions to memory performance in normal elderly. Brain Cogn 72, 400-407.

[49] Bonner-Jackson A, Mahmoud S, Miller J, Banks SJ (2015) Verbal and non-verbal memory and hippocampal volumes in a memory clinic population. Alzheimers Res Ther 7, 61 .

[50] Naveh-Benjamin M, Craik FI, Perretta JG, Tonev ST (2000) The effects of divided attention on encoding and retrieval processes: The resiliency of retrieval processes. $Q J \operatorname{Exp}$ Psychol A 53, 609-625.

[51] Sarter M, Bruno JP, Givens B (2003) Attentional functions of cortical cholinergic inputs: What does it mean for learning and memory? Neurobiol Learn Mem 80, 245-256.

[52] Petersen RC, Thomas RG, Grundman M, Bennett D, Doody R, Ferris S, Galasko D, Jin S, Kaye J, Levey A, Pfeiffer E, Sano M, van Dyck CH, Thal LJ, Alzheimer's Disease Cooperative Study Group (2005) Vitamin E and donepezil for the treatment of mild cognitive impairment. $N$ Engl $J$ Med 352, 2379-2388.

[53] Fitzpatrick-Lewis D, Warren R, Ali MU, Sherifali D, Raina P (2015) Treatment for mild cognitive impairment: A systematic review and meta-analysis. CMAJ Open 3, E419E427.

[54] Teipel SJ, Cavedo E, Grothe MJ, Lista S, Galluzzi S, Colliot O, Chupin M, Bakardjian H, Dormont D, Dubois B, Hampel H, Hippocampus Study Group (2016) Predictors of cognitive decline and treatment response in a clinical trial on suspected prodromal Alzheimer's disease. Neuropharmacology 108, 128-135.

[55] Dubois B, Chupin M, Hampel H, Lista S, Cavedo E, Croisile B, Louis Tisserand G, Touchon J, Bonafe A, Ousset PJ, Ait Ameur A, Rouaud O, Ricolfi F, Vighetto A, Pasquier F, Delmaire C, Ceccaldi M, Girard N, Dufouil C, Lehericy S, Tonelli I, Duveau F, Colliot O, Garnero L, Sarazin M, Dormont D, Hippocampus Study Group, Hippocampus Study 
Group (2015) Donepezil decreases annual rate of hippocampal atrophy in suspected prodromal Alzheimer's disease. Alzheimers Dement 11, 1041-1049.

[56] Martorana A, Mori F, Esposito Z, Kusayanagi H, Monteleone F, Codecà C, Sancesario G, Bernardi G, Koch G (2009) Dopamine modulates cholinergic cortical excitability in Alzheimer's disease patients. Neuropsychopharmacology 34, 2323-2328.
[57] Koch G, Di Lorenzo F, Bonní S, Giacobbe V, Bozzali M, Caltagirone C, Martorana A (2014) Dopaminergic modulation of cortical plasticity in Alzheimer's disease patients. Neuropsychopharmacology 39, 2654-2661.

[58] Mufson EJ, Ginsberg SD, Ikonomovic MD, DeKosky ST (2003) Human cholinergic basal forebrain: Chemoanatomy and neurologic dysfunction. J Chem Neuroanat 26, 233242 . 